\title{
HEREDABILIDADES Y TENDENCIAS GENÉTICAS PARA CARACTERÍSTICAS REPRODUCTIVAS EN UNA POBLACIÓN DE GANADO CRUZADO
}

\author{
HERITABILITY AND GENETIC TRENDS FOR REPRODUCTIVE TRAITS IN \\ CROSSBRED CATTLE POPULATION
}

\author{
OSCAR VERGARA G, ${ }^{\text {* }}$ Dr, RAFAEL CARDONA $R,{ }^{1} \mathrm{MVZ}$, DANIEL VALENCIA $\mathrm{H},{ }^{1} \mathrm{MVZ}$, \\ ROBERTO ALMANZA L, ${ }^{2}$ MV, CLARA RUGELES P, ${ }^{1}$ M.Sc.
}

${ }^{1}$ Universidad de Córdoba, Facultad de Medicina Veterinaria y Zootecnia, Grupo de Investigación en Producción Animal Tropical, Montería, Colombia. ${ }^{2}$ Gencaribe, Hacienda Abastecedora de carnes S.A. Planeta Rica, Córdoba, Colombia.

\section{Key words:}

Crossbreed, age at first calving, calving interval, breed effect.

\begin{abstract}
The objective of this study was to estimated genetic parameters and genetic trends for age at first calving (AFC), first calving interval ( $\mathrm{FCl}$ ), second calving interval ( $\mathrm{SCl}$ ), and third calving interval $(\mathrm{TCl})$ between the years 1999 at 2009 in a population of crossbred cattle in the municipality of Planeta Rica, Córdoba. Single trait models including the fixed effects of contemporary group (year-season-sex of calf, number calving), random additive direct genetic effects of the animal and residual was used. The estimation of variance components and genetic effects were estimated through AIREMLF90 program. Genetic trends were estimated as the basis of the average of the breeding values of animals per year through the Reg procedure of SAS. Heritability for direct additive genetic effects were $0.35 \pm 0.22$ for the $\mathrm{AFC}$, and $\mathrm{Cl} 1, \mathrm{Cl} 2$, and $\mathrm{Cl} 3$ were $0.16 \pm 0.03,0.003 \pm 0.046$, and $0.006 \pm 0.013$, respectively. The heritability estimate suggest that there is influence of the environment on the traits in study, and also should improve the conditions of feeding and management of animals. The estimated genetic trends averages tested positive and negative values near zero, indicating that the implementation of program of selection for these traits in the population is necessary.
\end{abstract}

\section{Resumen}

El objetivo de este estudio fue estimar parámetros genéticos y tendencias genéticas para edad al primer parto (EPP), primer intervalo entre partos (IEP1), segundo intervalo entre partos (IEP2) y tercer intervalo entre partos (IEP3) entre los años 1999 al 2009 en una población de ganado vacuno cruzado en el municipio de Planeta Rica, Córdoba. Se utilizaron modelos unicaracterísticos que incluyeron el efecto fijo del grupo contemporáneo (año-época-sexo de la cría-número de partos), efectos aleatorios genéticos aditivos directos del animal y residual. La estimación de los componentes de varianza y de efectos genéticos fueron estimados a través del programa AIREMLF90. Las tendencias genéticas se estimaron como regresión del promedio de los valores genéticos de los animales por año sobre el tiempo, mediante el procedimiento Reg de SAS. Las heredabilidades para los efectos genéticos aditivos directos fueron $0.35 \pm 0.22$ para la EPP, y para IEP1, IEP2 e IEP3 fue de; $0.16 \pm 0.03,0.003$ \pm 0.046 y $0.006 \pm 0.013$, respectivamente. Los estimados de heredabilidad sugieren que hay influencia del ambiente sobre las características en estudio y que además se deben mejorar las condiciones de alimentación y manejo de los animales. Las tendencias genéticas estimadas fueron cercanas a cero, lo cual indica que se hace necesario la implementación de un programa de selección para estas características en la población. 


\section{Introducción}

Las características reproductivas como el intervalo entre parto (IEP) y la edad al primer parto (EPP) son aspectos importantes que determinan la rentabilidad de una empresa ganadera y determinadas por múltiples factores como la nutrición, genética, clima, sanidad y manejo (CASAS y TEWOLDE, 2001).

Las características reproductivas son importantes para todos los sistemas de producción pecuarios y debe ser consideradas dentro de los objetivos de un programa de mejoramiento genético, a pesar de presentar valores bajos de heredabilidad y de la dificultad que se presenta en la recolección de datos (BORMANN y WILSON, 2010; BARROZO et al., 2012)

Es evidente que existe una alta variabilidad entre razas para la eficiencia reproductiva, ya sea bajo condiciones tropicales o climas templados. En climas templados abundan las razas de origen Bos taurus utilizadas en la producción de carne bovina. En climas tropicales la producción de carne es principalmente a base de razas de origen Bos indicus, o cruzamientos entre Bos taurus y Bos indicus. En los ecosistemas tropicales el uso masivo de las razas Bos indicus ha reflejado baja eficiencia productiva, por lo que su impacto ha sido limitado (MORENO, 2002).

Por lo anterior, para mejorar la edad al primer parto y los intervalos entre partos es importante para las empresas ganaderas conocer sus parámetros genéticos, por lo que objetivo del presente trabajo fue estimar la heredabilidad y tendencias genéticas de las características reproductivas en ganado cruzado en un sistema de producción de carne en la zona del trópico bajo de Colombia, del departamento de Córdoba.

\section{Materiales y métodos}

Localización. El estudio se realizó en una hacienda ganadera localizada en el municipio de Planeta Rica, departamento de Córdoba. Este Municipio se encuentra a una altura de 100 m.s.n.m., con una temperatura promedio de $34^{\circ} \mathrm{C}$ y humedad relativa de $85 \%$. Está ubicado a $8^{\circ} 17^{\prime} 15^{\prime \prime}$ Latitud Norte y $75^{\circ} 73^{\prime} 55^{\prime \prime}$ Longitud Oeste. La hacienda cuenta con un total de 2000 animales y 1350 hectáreas divididas en cuatro fincas, el manejo nutricional es a base de pastos como Braquiaria (Brachiaria decumbens), Humidicola (Brachiaria humidicola), Angletón (Dichantium aristatum), Estrella Africana (Cynodon nlemfuensis), Admirable (Brachiaria mutica), Llanero (Brachiaria Dictyoneura) y en los periodos críticos se suministra heno de Dichantium aristatum y silo de maíz (Zea mays). Además, a los animales se les proporciona sal y agua a voluntad.
Animales y datos. Se tomó la información de los registros productivos y reproductivos entre los años 1999 y 2009 de los grupos genéticos Brahman (B), Cebú comercial (CC), F1 Angus x Brahman (F1AB) y 3/4 Brahman x 1/4 Angus (3/4B1/4A). El número de datos para cada grupo genético se presenta en la Tabla 1 . Para la edad al primer parto se utilizaron 334 registros, para el primero, segundo y tercer intervalo de parto se utilizaron 297, 230 y 145 registros, respectivamente (Tabla 2). Cabe resaltar que el bajo número de registros para las características evaluadas, se debe a que la información proviene de un solo rebaño y a la limitante en la toma de información por parte de los productores en los sistemas de producción del trópico bajo colombiano.

Tabla 1. Número de animales por grupo genético para cada una de las características evaluadas.

\begin{tabular}{ccccc}
\hline Grupo genético & EPP & IEP1 & IEP2 & IEP3 \\
\hline Brahman & 231 & 206 & 154 & 90 \\
Cebú comercial & 34 & 30 & 27 & 25 \\
F1 Brahman x Angus & 42 & 36 & 33 & 30 \\
$3 / 4$ Brahman + 1/4 Angus & 27 & 25 & 16 & No \\
\hline
\end{tabular}

Para la depuración de la base de datos se utilizaron varios procedimientos del Statistical Analysis System (SAS, 2006). En la genealogía de EEP se utilizaron 473 animales, para IEP1 428, para IEP2 340 y para IEP3 220 animales.

Parámetros genéticos y predicciones genéticas. Los datos fueron analizados mediante modelos unicarácter, los componentes de varianza y covarianza se calcularon utilizando el procedimiento de Máxima Verosimilitud Restringida. Para ello, se utilizó el software AIREMLF90 (MISZTAL, 1997; TSURUTA, 1999), el cual utiliza algoritmo de información promedio. El modelo estadístico usado fue el siguiente:

$y_{i j k}=G_{i}+R_{j}+A_{k}+e_{i j k}$, donde:

$\mathrm{y}_{\mathrm{ikk}}=$ valor observado para EPP, IEP1, IEP2 o IEP3;

$\mathrm{G}_{\mathrm{i}}=$ efectos fijo de grupo contemporáneo: año - época sexo de la cría, número de partos, donde el año varió de 1999 al 2009, la época varió de 1 (seca: diciembre a abril) a 2 (lluvia: mayo a noviembre), el sexo varió de 1(macho) a 2 (hembra).

$\mathrm{R}_{\mathrm{j}}=$ efecto fijo del j-ésimo grupo racial del animal (Brahman; Cebú comercial; Angus x Brahman; 3/4 Brahman x 1/4 Angus).

$A_{k}=$ efecto genético aleatorio del k-ésimo animal.

$\mathrm{e}_{\mathrm{ijk}}=$ efecto residual aleatorio.

Las tendencias genéticas se calcularon como una regresión lineal de las medias ponderadas de los valores genéticos sobre los respectivos años, usando el procedimiento REG de Statistical Analysis System (SAS, 2006). 


\section{Resultados}

Los promedios para las características evaluadas se presentan en la Tabla 2. Las heredabilidades estimadas fueron $0,35 \pm 0,22$ para EPP, $0,16 \pm 0,03$ para IEP1, $0,003 \pm 0,046$ para IEP2 y 0,006 $\pm 0,013$ para IEP3. Los promedios de las características evaluadas se presentan en la Tabla 2.

Tabla 2. Promedios para la edad al primer parto e Intervalos entre partos.

\begin{tabular}{ccccc}
\hline Parámetro & EPP & IEP1 & IEP2 & IEP3 \\
\hline N & 334 & 297 & 230 & 145 \\
Promedio (días) & 1143 & 532 & 525 & 506 \\
Desviación estándar (días) & 144 & 94 & 75 & 62,4 \\
Coeficiente de variación (\%) & 12,6 & 17,7 & 14,3 & 12,3 \\
\hline
\end{tabular}

Las tendencias genéticas para los años de estudio de EPP, IEP1, IEP2 e IEP3 se observan en las Figs. 1, 2,3 y 4 .

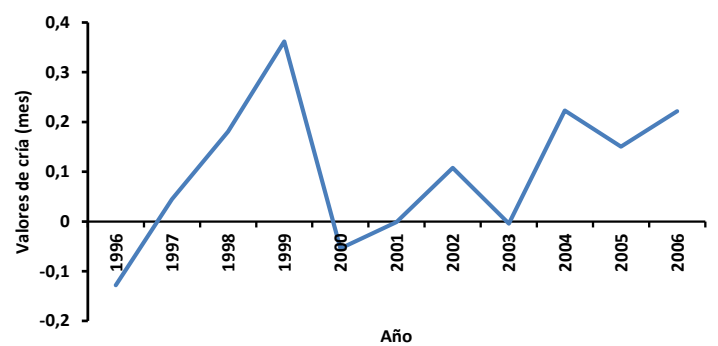

Figura 1. Tendencias geneticas para edad al primer parto.

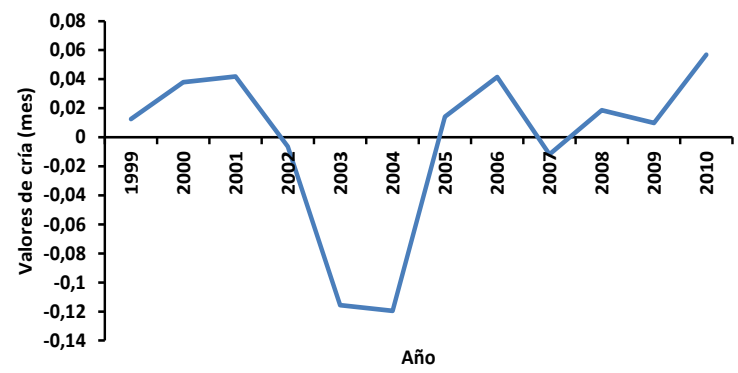

Figura 2. Tendencias geneticas para el primer intervalo entre partos.

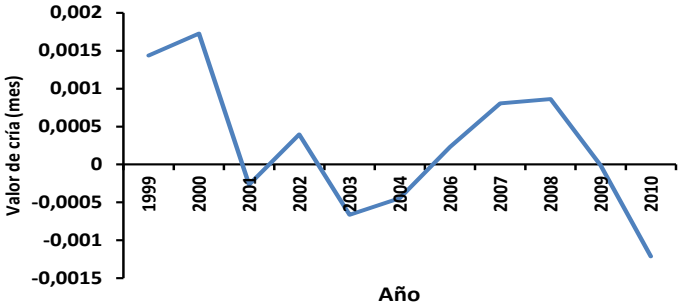

Figura 3. Tendencias geneticas para el segundo intrevalo entre partos.

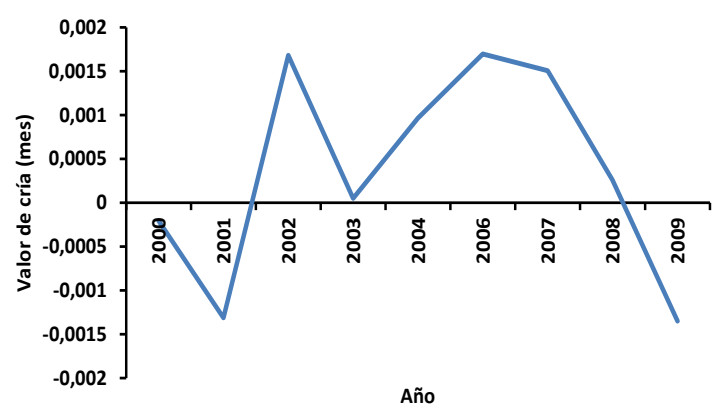

Figura 4. Tendencias genéticas para el tercer intervalo entre partos

En la Tabla 3 se pueden observar las ecuaciones de regresión de las tendencias genéticas para las características estudiadas. Se puede notar que ninguna de las tendencias fue significativa $(P>0.05)$.

Tabla 3. Regresiones lineales de las tendencias genéticas (mes/año) de las características reproductivas evaluadas

\begin{tabular}{cccc}
\hline Característica & Ecuación de regresión & Error estándar & $\boldsymbol{P}>\boldsymbol{F}$ \\
\hline EPP & $\mathrm{y}=-31,37+0.0157 \mathrm{x}$ & 0.0135 & 0.2739 \\
IEP1 & $\mathrm{y}=-4,55+0,0023 \mathrm{x}$ & 0,0050 & 0,6608 \\
IEP2 & $\mathrm{Y}=0,22-0,0001 \mathrm{x}$ & 0,0001 & 0,1512 \\
IEP3 & $\mathrm{Y}=-0,05+0,00003 \mathrm{x}$ & 0,0001 & 0,8616 \\
\hline
\end{tabular}

En la Tabla 4 se presentan las soluciones para los efectos raciales de las características evaluadas. Las soluciones para EPP fueron desviadas de Brahman y las soluciones para IEP1, IEP2 e IEP3 se desviaron respecto a Cebú comercial.

Tabla 4. Soluciones ( \pm error estándar) para los efectos de grupo genéticos de EPP (desviado de Brahman) e IEP 1, 2 y 3 (desviados de Cebú comercial).

\begin{tabular}{ccccc}
\hline \multirow{2}{*}{ Grupo genético } & \multicolumn{4}{c}{ Característica } \\
\cline { 2 - 5 } & EPP & IEP1 & IEP2 & IEP3 \\
\hline \multirow{2}{*}{ Brahman } & 0,000 & 1.148 & 0.590 & 0.836 \\
& $\pm 0,000$ & \pm 0.660 & \pm 0.535 & \pm 0.560 \\
Cebú comercial & $-0,828$ & 0.000 & 0.000 & 0.000 \\
& $\pm 0,780$ & \pm 0.000 & \pm 0.000 & \pm 0.000 \\
F1 Angus x Brahman & $-4,384$ & 0.073 & -0.238 & 0.758 \\
& $\pm 0,876$ & \pm 0.877 & \pm 0.651 & \pm 0.661 \\
3/4 Brahman x 1/4 Angus & 0,858 & 0.430 & -0.254 & \\
& $\pm 0,948$ & \pm 0.933 & \pm 0.896 & \\
\hline
\end{tabular}

\section{Discusión}

Edad al primer parto. La media para la EPP fue similar a la reportada por VERGARA et al. (2016) en la raza Romosinuano $(1158 \pm 286)$ y por CASAS y TEWOLDE (2001) y GARCÍA et al. (2003), en hembras Romosinuanas $(1146 \pm 293)$ y en Brahman, Neloré, 
Guzerat, y Cebú comercial (1171), respectivamente. El resultado obtenido en este trabajo fue superior al reportado por ESTRADA-LEÖN et al. (2008), en la raza Brahmán (1091士147), BOLIGON et al. (2010), en vacas de Nelore (1050 \pm 109$)$ y por MARTíNEZ et al. (2009) en vacas Sanmartineras $(989 \pm 190)$. Valores superiores fueron reportados por VERGARA et al. (2009), en una población cruzada de Cebú Comercial, Pardo Suizo, Holstein, Simmental y Sahiwal $(1186 \pm 119$ días $)$ y por VERGARA et al. (2015) en una población AngusBrahman. La EPP obtenida en este trabajo se considera adecuada de acuerdo a las condiciones del trópico bajo colombiano, pero se podría mejorar, proporcionando a los animales un buen manejo reproductivo al momento de entrar las novillas a servicio.

La heredabilidad para la EPP fue muy similar a la reportada por MARTÍNEZ et al. (2009), en la raza criolla Sanmartinero, BERRY y EVANS (2014) en ganado cruzado y VERGARA et al. (2015) en ganado cruzado $(0,34 \pm 0,22,0,31 \pm 0,02$ y $0.32 \pm 0.09$, respectivamente). La heredabilidad encontrada en este trabajo fue superior a las reportadas por OSSA et al. (2008) en hembras de la raza Romosinuano $(0,05$ $\pm 0,04)$ y a la encontrada por BOLIGON et al. (2010) y CAETANO et al. (2013) en ganado Nelore $(0,17 \pm$ 0,01 y $0,17 \pm 0,02$, respectivamente). Por su parte, ESTRADA-LEÓN et al. (2008) en ganado cebuino, estimaron una heredabilidad mayor a la encontrada en este estudio $(0,46 \pm 0,14)$. El valor estimado de heredabilidad para la EPP de este estudio se considera moderado, por lo que a través de la selección se podría lograr progreso genético para este carácter. Además, se requiere mejorar la alimentación y el manejo de los animales para que ellos expresen completamente su potencial genético para esta característica. Hay que considerar que el error estándar de la heredabilidad para los caracteres evaluados es alto, debido al número de datos considerados en la estimación.

La tendencia genética para EPP presenta un comportamiento variable en el transcurso de los años evaluados (Fig. 1) y fue no significativa y cercana a cero (Tabla 3 ), esto sugiere que no hubo progreso genético, por lo se hace necesario la implementación de un programa de selección para esta característica en la población. Aunque, a partir del año 2000 se aprecia un incremento en los valores genéticos en el tiempo, por lo que habría que determinar esta tendencia en evaluaciones futuras.

Intervalos entre partos. La media estimada para el IEP1 (Tabla 2) fue superior a la reportada por PEROTTO et al. (2006), en un grupo racial Guzerat x Nelore, OSSA et al. (2007) en vacas de las raza Romosinuano y VERGARA et al. (2016) en ganado Romosinuano (505 \pm $25,433,60 \pm 139,18$ y $409 \pm 88$, días respectivamente).
Por su parte, GOSHU et al. (2014) estimaron un promedio similar al de este estudio $(521 \pm 5,8)$.

La media estimada para IEP2 fue superior a las reportados por PEROTTO et al. (2006), en la raza Nelore (492 \pm 22 ) y los cruces Nelore x Guzerat (505 \pm 25$)$, Nelore $x$ Red Angus (433 \pm 22 ), Nelore $x$ Marchigiana (453 \pm 27 ) y Nelore $x$ Simental (450 \pm 24$)$. MOTTA et al. (2012), también reportaron un IEP2 más corto en ganado F1 Gyr x Holstein $(441 \pm 87)$ en clima cálido colombiano. Así mismo, LÓPEZ et al. (2010), en ganado Bos taurus x Bos inidicus y HERNÁNDEZ et al. (2000), en ganado doble propósito reportaron medias más bajas respecto a la de este estudio (465 \pm 9.3 y $447 \pm 10$ días, respectivamente).

La media encontrada en este trabajo para el IEP3 fue de $506 \pm 62$ días, valor superior a las medias reportadas HERNÁNDEZ et al. (2000) en ganado doble propósito (433 \pm 10$)$, LÓPEZ et al. (2010), en ganado Bos taurus $x$ Bos inidicus $(452 \pm 11)$ y MAGAÑA et al. (2002) en ganado Cebú (450 \pm 8$)$.

De acuerdo a los valores estimados para IEP1, IEP2 y IEP3, se debería mejorar el manejo reproductivo de los animales, con el fin de disminuir los promedios de estas características. Además, se puede notar que el menor intervalo entre partos fue el IEP3, debido seguramente a que en este periodo la vaca ya está completamente desarrollada tanto anatómica como fisiológicamente; y el mayor intervalo fue IEP1, periodo en el cual la vaca aún está en proceso de crecimiento. De acuerdo a lo anterior, al momento de tomar decisiones respecto a los intervalos entre partos, hay que tener en cuenta la edad de la vacas y que estos son afectados por factores ambientales y genéticos (NOGUEIRA, 2004).

El estimado de heredabilidad obtenido en este estudio para IEP1 fue similar a los reportados por VERGARA et al. (2009) en una población multirracial de Angus, BON y Cebú en Colombia $(0,15 \pm 0,13)$, ESTRADA-LEÓN et al. (2008) en ganado Brahman $(0,11 \pm 0,06)$ y DUITAMA et al. (2013), en vacas Brahman $(0,11 \pm 0,03)$. Valores inferiores fueron hallados por FARAJI et al. (2011) en ganado de la raza Holstein $(0.04 \pm 0,003)$ y VERGARA et al. (2016) en la raza Romosinuano $(0,06 \pm 0,06)$.

Para el IEP2, la heredabilidad estimada fue inferior a la reportada por VERGARA et al. (2009) en una población multirracial $(0,18 \pm 0,11)$, por FARAJl et al. (2011) en ganado de la raza Holstein $(0,03 \pm 0,004)$, por VERGARA et al. (2015) en ganado cruzado Angus $x$ Brahman $(0,14 \pm 0,05)$ y por VERGARA et al. (2016) en ganado Romosinuano $(0,09 \pm 0,06)$. En la literatura consultada no se encontraron reportes de IEP3 para ganado vacuno. De acuerdo a las heredabilidades estimadas para los diferentes intervalos, se recomienda 
mejorar la alimentación de vacas y novillas y el periodo periparto, ya que esto puede tener gran influencia sobre los intervalos entre partos (VERGARA et al., 2009). Además, como estas característica están principalmente controladas por efectos ambientales y genéticos no aditivos, no se espera que haya una buena respuesta a la selección para estas características (LAUREANO et al., 2011; ROCHA et al., 2012).

Por otra parte, las tendencias genéticas para IEP1, IEP2 e IEP3 fueron no significativas y cercanas a cero (Tabla 3). Los resultados de las tendencias genéticas para las características estudiadas durante el periodo de la evaluación, sugieren que no hubo progreso genético para estas características, lo que requiere la implementación de un programa de selección si se desean mejorar los intervalos entre partos. De acuerdo a los valores de las tendencias genéticas y los promedios de los intervalos entre partos, en el sistema de producción se puede estar incrementando el intervalo generacional, lo cual disminuiría su rentabilidad.

Grupos genéticos. Para los efectos raciales, se encontró que los animales Brahman tuvieron una EPP mayor que los Cebú comercial y los F1 Angus $x$ Brahman, pero una EPP menor que los animales con proporciones $3 / 4$ Brahman $\times 1 / 4$ Angus. Esto sugiere, que animales Cebú comercial tiene una EPP de -25 días, y que el F1 Angus x Brahman fue aún más precoz con -134 días para la EPP en comparación con el Brahman. FACÓ et al. (2005) reportaron una EPP para cinco grupos genéticos de ganado Holandés x Gyr en Brasil, donde el grupo genético del F1 fue el que presentó una edad más baja al primer parto, tal como sucedió para el F1 en este trabajo.
Para el IEP1, Brahman, F1 Angus x Brahman y $3 / 4$ Brahman $\times 1 / 4$ Angus tuvieron un mayor IEP1 respecto al Cebú comercial. Para el IEP2, 3/4 Brahman x $1 / 4$ Angus y $\mathrm{F} 1$ Angus $x$ Brahman tuvieron un IEP2 menor en 8 y 7 días, respecto al Cebú comercial. Por el contrario, Brahman tuvo un IEP2 de 18 días por encima al estimado para el Cebú comercial. Para el IEP3, F1 Angus $x$ Brahman y Brahman tuvieron un intervalo entre partos de 23 y 25 días, respectivamente, por encima del Cebú comercial. Cabe resaltar que para el grupo genético $3 / 4$ Brahman $\times 1 / 4$ Angus no hubo valores estimados, debido a que el número de animales evaluados para esta característica era muy pequeño. De acuerdo a lo anterior, para la EPP e IEP1, IEP2 e IEP3 de los grupos genéticos evaluados, podemos decir que el $\mathrm{F} 1$ Angus $x$ Brahman fue el que mejor desempeño mostró respecto a los otros grupos raciales. Según NOGUEIRA et al. (2004), esto se debe a que los cruces de Cebú $x$ Bos taurus presentan una mejor heterosis en relación con la edad de la pubertad.

\section{Conclusión}

De acuerdo a los resultados obtenidos encontrados se puede afirmar que la media obtenida para la EPP es buena, sin embargo, los IEP fueron amplios, por lo que se bebe mejorar las condiciones de alimentación en el periodo peri y posparto, con el fin de mejorar la eficiencia reproductiva del ganado cruzado del hato. Las heredabilidades estimadas para la EPP, IEP1, IEP2 e IEP3 están entre bajas y medias, lo cual sugiere que para realizar una mejora genética a estos parámetros el proceso será lento en esta población de ganado cruzado. Los valores de tendencias genéticas para las características estudiadas sugieren que se debe implementar un programa de selección en la población.

\section{Referencias}

BARROZO, D.; BUZANSKAS, M.; OLIVEIRA, J.; MUNARI, D.; NEVES, H.; QUEIROZ, S. 2012. Genetic parameters and environmental effects on temperament score and reproductive traits of Nellore cattle. Animal 6(1): 36-40.

BERRY, D. P.; EVANS, R. D. 2014. Genetics of reproductive performance in seasonal calving beef cows and its association with performance traits. Journal of Animal Science 92(4): 1412-1422.

BOLIGON, A.; DE ALBUQUERQUE2, L.; MERCADANTE, M.; LÔBO, R. 2010. Study of relations among age at first calving, average weight gains and weights from weaning to maturity in Nellore cattle. Revista Brasileira de Zootecnia 39(4): 746-751.

BORMANN, J. M.; WILSON, D.E. 2010. Calving day and age at first calving in Angus heifers. Journal of Animal Science 88: 1947-1956.

CAETANO, S. L.; SAVEGNAGO, R. P.; BOLIGON, A. A.; RAMOS, S.B.; CHUD, T. C.; LÔBO, R. B.; MUNARI, D. P. 2013. Estimates of genetic parameters for carcass, growth and reproductive traits in Nellore cattle. Livestock Science 155(1): 1-7. 
CASAS, E.; TEWOLDE, A. 2001. Evaluación de características relacionadas con la eficiencia reproductiva de genotipos criollos de carne en el trópico húmedo. Archivo Latinoamericano Producción Animal 9(2): 68-73.

DUITAMA, O. C.; GONZÁLES, L. H.; GARCIA, D.; FARAH, M.; DA FONSECA, R. 2013. Productividad acumulada y su relación genética con características reproductivas en hembras Brahman. Revista MVZ Córdoba 18(Supl): 3658-3664.

ESTRADA-LEÓN, R.; MAGAÑA, J.; SEGURA-CORREA, J. 2008. Parámetros genéticos para caracteres reproductivos de vacas Brahman en un hato del sureste de México. Tropical and Subtropical Agroecosystems 8(3): $259-263$.

FACÓ, O.; BRAGA, R.; FILHO, R.; LIMA, F. 2005. Idade ao primeiro parto e intervalo de partos de cinco grupos genéticos Holandês x Gir no Brasil. Revista Brasileira de Zootecnia 34(6): 1920-1926.

FARAJI-AROUGH, H.; ASGHAR, A.; FARHANGFAR, H. 2011. Estimation of genetic parameters and trends for age at first calving and calving interval in Iranian Holstein cows. Journal of Research in Agricultural Science 7(1): 79-87.

GARCÍA, G.; MALDONADO-ESTRADA, J.; LÓPEZ, J. 2003. Caracterización productiva y reproductiva de las explotaciones ganaderas del bajo cauca y el litoral atlántico antioqueños. II. Comportamiento de cuatro grupos raciales Bos indicus en un sistema de bosque seco tropical (bs-T). Revista Colombiana de Ciencias Pecuarias 16(2): 117-125.

GOSHU, G.; SINGH, H.; PETERSSON, K.; LUNDEHEIM, N. 2014. Heritability and correlation among first lactation traits in Holstein Friesian cows at Holeta Bull Dam Station, Ethiopia. International Journal of Livestock Production 5(3): 47-53.

HERNÁNDEZ, E.; SEGURA, V.; SEGURA, J.; OSORIO, M. 2000. Intervalo entre partos, duración de la lactancia y producción de leche un hato de doble proposito en Yucatan, México. Agrociencia 34(6): 699-705.

LAUREANO, M.; BOLIGON, A.; COSTA, R.; FORNI, S.; SEVERO, J. 2011. Estimativas de herdabilidade e tendências genéticas para características de crescimento e reprodutivas em bovinos da raça Nelore. Arquivos Brasileiros de Medicina Veterinária e Zootecnia 63(1): 143-152.

LÓPEZ, R.; HERNÁNDEZ, M.; GARCÍA, J.; NÚŃEZ, R.; ORDAZB, R.; MARTÍNEZ, M. 2010. Eventos reproductivos de vacas con diferente porcentaje de genes Bos taurus en el trópico mexicano. Revista Mexicana de Ciencias Pecuarias 1(4): 325-336.

MAGAÑA, J.; DELGADO, R.; SEGURA, J. 2002. Factores ambientales y genéticos que influyen en el intervalo entre partos y el peso al nacer del ganado Cebú en el sureste de México. Revista Cubana de Ciencia Agrícola 36(4): 317-322.

MARTINEZ, G.; MARTÍNEZ, G.; MANRIQUE, C. 2009. Estimación de parámetros genéticos de edad al primer parto e intervalo entre partos de vacas criollas Sanmartineras (SM). Revista Orinoquia 13(2): 113-125.

MISZTAL, I. BLUPF90 - a flexible mixed model program in Fortran 90. University of Georgia [serial online] 1997 [citado 26 Oct 2015]. Disponible en: URL: http://nce.ads.uga.edu/html/projects/blupf90.pdf.

MORENO, F. 2002. Diversidad genética y relaciones filogenéticas del ganado criollo Colombiano. Revista CORPOICA 3: 17-25.

MOTTA, P.; RIVERA, L.; MARIÑO, A.; LIZCANO, C. 2012. Desempeño productivo y reproductivo de vacas F1 Gyr $\mathrm{x}$ Holstein en clima cálido colombiano. Veterinaria y Zootecnia 6(1): 17-23.

NOGUEIRA, G. P. 2004. Puberty in South American Bos indicus (Zebu) cattle. Animal Reproduction Science 82-83: 361-372.

OSSA, G.; SUÁREZ, M.; PÉREZ, J. 2007. Factores ambientales y genéticos que influyen la edad al primer parto y el intervalo entre partos en hembras de la raza criolla Romosinuano. Revista CORPOICA 8(2): 74-80. 
OSSA, G.; PÉREZ, J.; SUÁREZ, M. 2008. Valores genéticos de caracteres productivos y reproductivos en bovinos Romosinuano Cereté: Revista CORPOICA 9(1): 93-101.

PEROTTO, D.; SANTOS, J.; KROETZ, I. 2006. Intervalo de partos de fêmeas bovinas Nelore, Guzerá x Nelore, Red Angus x Nelore, Marchigiana x Nelore e Simental x Nelore. Revista Brasileira de Zootecnia 35(3): 733-741.

ROCHA, J.; GALLEGO, J.; VÁSQUEZ, R.; PEDRAZA, J.; ECHEVERRI J.; CERÓN-MUÑOZ, M.; MARTÍNEZ, R. 2012. Estimación de parámetros genéticos para edad al primer parto e intervalo entre partos en poblaciones bovinas de la raza Blanco Orejinegro (BON) en Colombia. Revista Colombiana de Ciencias Pecuarias. 25: $220-228$.

STATISTICALANALYSIS SYSTEM INSTITUTE (SAS) 2006 SAS/STAT User's guide (Relase 9.1.3), Cary, NC, USA.

TSURUTA, S. A modification of REMLF90 with computing by the Average-Information Algorithm. University of Georgia [serial online] 1999 [citado 26 Oct 2015]. Disponible en: URL: http://nce.ads.uga.edu/wiki/doku.php?id=readme.aireml.

VERGARA, O.; BOTERO, L.; MARTÍNEZ, C. 2009. Factores ambientales que afectan la edad al primer parto y primer intervalo de partos en vacas del sistema doble proposito. Revista MVZ Córdoba 14(1): 1594-1601.

VERGARA, O.; ELZO, M.; CERÓN-MUÑOZ, M. 2009. Genetic parameters and genetic trends for age at first calving and calving interval in an Angus-Blanco Orejinegro-Zebu multibreed cattle population in Colombia. Livestock Science 126: 318-322.

VERGARA, O.; MARTÍNEZ, N.; FLÓREZ, M.; HERNÁNDEZ, M.; ALMANZA, R.; RÚGELES, C.; SIMANCA, J. 2015. Heredabilidades, correlaciones y tendencias genéticas para características reproductivas en una población bovina multirracial en Colombia. Revista CES Medicina Veterinaria y Zootecnia 10(1): 8-17.

VERGARA, O.; OSSA, G.; CABRERA, J.; SIMANCA, J.; PÉREZ, J. 2016. Heritabilities and genetic trends for reproductive traits in a population of Romosinuano cattle in Colombia. Revista MVZ Córdoba 21(1): 5250-5257. 